\title{
Emperadores y cristianos en el siglo III
}

\author{
Narciso Santos Yanguas*
}

\section{INTRODUCCIÓN}

Continuando con el estudio y análisis que hemos venido realizando en los últimos años acerca de las relaciones establecidas, por un lado entre la religión cristiana y los personajes más representativos de la Iglesia (obispos y clérigos en general, sin olvidar la situación concreta de las diferentes comunidades de fieles, adeptos y practicantes de dichas creencias), y por otro la administración romana, junto con sus miembros institucionales más significados (emperadores, gobernadores provinciales, legados imperiales...), nos disponemos a abordar a continuación de forma general las características de dicho conflicto a lo largo del siglo III de nuestra era ${ }^{1}$.

De la misma manera que hemos analizado con respecto a los períodos históricos anteriores, nuestro objetivo se va a centrar en desentrañar no sólo el comportamiento de los emperadores correspondientes a dicha época (en especial algunos de ellos), sino también en el estudio y significado de los decretos y rescriptos imperiales emitidos con relación al cristianismo y que, en última instancia, condicionarian la línea de actuación legal con referencia a la nueva religión?

* Universidad de Oviedo.

1 Para las dos centurias precedentes ver N. SANTOS, Cristianismo e Imperio romano durante el siglo I, Madrid, 1994 (2. edic.) y Cristianismo e Imperio romano durante el siglo 1 , Madrid, 1996.

2 Analizaremos sucesivamente el contexto en el que se inscriben las normas emanadas a este respecto de las cancillerías imperiales durante las dinastías de los Severos, así como las correspondientes a Maximino el Tracio, Decio y Valeriano sobre todo, antes de estudiar el alcance y los parámetros que encierra el reconocimiento de la Iglesia por parte de Galieno. 
En este sentido no podemos olvidar un hecho que tiene sus raíces ya en las décadas finales de la primera centuria de nuestra era, pero que se iría acentuando a medida que nos adentramos en el siglo II: la presión ejercida por las masas populares paganas que habitaban los centros urbanos sobre las comunidades cristianas enraizadas en dichos enclaves, así como sobre sus lugares de reunión y asambleas (iglesias, capillas...) y los centros de enterramiento de sus muertos (cementerios).

Pero si este elemento sigue vigente aún en la etapa histórica que nos proponemos analizar hay otro que pierde casi todo el sentido que había adquirido a lo largo de la centuria precedente, el constituido por el culto imperial ${ }^{3}$.

A este respecto las ceremonias vinculadas a dicho culto, así como el sentido, alcance y significado del mismo, prácticamente se irían diluyendo a lo largo del siglo III, tal vez porque el valor dinástico que estaba implícito en el mismo iría desapareciendo igualmente como consecuencia de las turbulencias políticas y las sucesiones de los emperadores a lo largo de dichas décadas a manos de los distintos ejércitos romanos, hasta el extremo de no contar ya con un punto referencial concreto, de forma muy distinta a lo que había sucedido por ejemplo durante la dinastía de los Antoninos en el transcurso de la segunda centuria de nuestra era 4 .

Por consiguiente se hace preciso pasar revista en todo caso al conjunto de órdenes legales y jurídicas (edictos y rescriptos imperiales) que, procedentes de la administración central romana a través del emperador o de los miembros de su consejo (incluidos los mejores jurisconsultos de la época), iban a desencadenar una línea de actuación con respecto a los cristianos y a sus diferentes comunidades, a medida que la iniciativa de las masas populares, puesta de manifiesto a través de denuncias y tumultos de carácter público, perdería su importancia.

Ahora bien, esta legislación anticristiana, que tiene sus primeras manifestaciones ya en las últimas décadas del siglo I de nuestra era ${ }^{5}$, termi-

\footnotetext{
3 Para más detalles ver N. SANTOS, "Presión popular y culto al emperador en las persecuciones anticristianas del siglo II», HAnt 18 (1994), 305 y ss.

4 Sólo parcialmente durante el primer tercio del siglo ill la dinastía de los Severos (años 193 a 235) lograría preservar algunos de los aspectos vinculados al culto imperial, a pesar de que el origen oriental de muchos de los emperadores y la introducción de los cultos mistéricos en el panteón romano desvirtuarían las características primitivas del mismo. Cf. R. TURCÁN, «Le culte impérial au Ille siècle», ANRW, 2.16 .2 (1978), 996 y ss.

5 Ver, como ejemplo, T.D. BARNES, "Legislation against the Christians", JRS, 58 (1968), 32 y ss., y S. Prete (a cura di), Cristianesimo e Impero romano. Base giuridica dalle persecuzioni. Antologia critica, Bolonia, 1974.
} 
naría emergiendo casi exclusivamente del ámbito de la administración al haberse convertido el colectivo de los cristianos, desde el punto de vista del Estado romano, en una organización de tipo criminal.

Por otro lado, hemos de añadir el hecho de que la Iglesia cristiana había ido adquiriendo simultáneamente, desde esa misma perspectiva del Estado romano, una entidad tan fuerte que podía llegar a constituir un cierto peligro frente a los intereses generales del Imperio.

En este contexto va a ser posible descubrir un contraste acusado entre la primera y la segunda mitad del siglo III en cuanto a la legislación anticristiana, estando configurado el momento de inflexión por la etapa correspondiente al emperador Decio en los comedios de dicha centuria.

De cualquier forma la organización de las comunidades cristianas había ido adquiriendo una importancia tan grande en el seno de los ambientes municipales que tan amplio desarrollo conduciría inexorablemente a su consideración como peligrosas, poniendo en estado de alerta a buena parte de las autoridades públicas de la administración romana ${ }^{6}$.

Como consecuencia del nuevo planteamiento algunos de los emperadores romanos de la segunda mitad de dicha centuria (en especial Decio y Valeriano) van a poner todo su empeño en conseguir derrotar al cristianismo y a los medios de infraestructura que sustentaban la nueva religión, no sólo desde el punto de vista de la confiscación de sus lugares de culto y reunión sino también a través de la persecución de quienes se habían esforzado en el proselitismo cristiano, así como en los neófitos de la nueva doctrina.

A pesar de todo el giro de los acontecimientos variaría sustancialmente, dado que la gran mayoría de la población pagana, especialmente los habitantes de los núcleos urbanos: no iban a hacer caso ya durante mucho tiempo del cúmulo de absurdas calumnias que se venían formulando de forma tradicional contra el colectivo de los cristianos?

6 En otros términos, el poder de los cristianos había adquirido tal pujanza y vigor, incluida su participación en los órganos de gobierno romano, tanto de la administración central como provincial o local, que las autoridades romanas, encabezadas por el propio emperador, se verían en la necesidad de cercenar dicho crecimiento e incluso, si era posible, acabar con él.

7 Lo cual no impedía que los propios emperadores se esforzaran, en ocasiones con verdadera desesperación, por revitalizarlas con todos los medios a su alcance. Cf., por ejemplo, V.A. Sirago, Involuzione politice e spirituale nell impero del il secolo, Nápoles, 1974. 
Así pues se trata de concretar el grado de aplicación de los edictos imperiales en los que se hallaban implicadas las comunidades cristianas, aunque ello no suponga que nos vayamos a detener de forma monográfica en todos y cada uno de los casos particulares de acusación y martirio a los que pudieron haber dado origen ${ }^{8}$.

\section{CARÁCTER DE LA DOCUMENTACIÓN}

La realidad socio-política y religiosa que supone el contacto entre cristianismo e Imperio romano durante el siglo II, incluidos los brotes de persecución más notables contra los adeptos de dichos cultos, aparece recogida en una serie de fuentes de información antiguas; algunas de ellas son obra de autores vinculados a la nueva religión, mientras que otras referencias nos han sido transmitidas por escritores paganos, por lo que resulta necesario analizar si realmente confluyen las noticias de unos y otros, dadas las perspectivas de análisis tan dispares que podemos observar a primera vista en las apreciaciones de los mismos ${ }^{9}$.

La documentación de los autores paganos con relación a la situación del cristianismo en el marco del Imperio romano a lo largo de dicha centuria aparece reseñada, por lo general de forma muy escueta, en los llamados Escritores de la Historia Augusta; estos autores nos ofrecen pinceladas acerca de la consideración de dicha doctrina por parte de los emperadores de la dinastía de los Severos y posteriormente por alguno de los pertenecientes al período convulsivo de la historia romana conocido como Anarquía militar ${ }^{10}$.

En este contexto pagano sobresalen en primer término los llamados libelos (libelli), cuyo sentido más completo se corresponde con la etapa del emperador Decio ${ }^{11}$, a pesar de que su origen y lo que significaban desde

8 Para completar estos aspectos remitimos, entre otros, a H. Musurillo, Acta Martyrum. The Acts of the Christian Martyrs, Oxford 1972, y D. Ruiz BuEno, Actas de los mártires, Madrid, 1974 (3. ${ }^{\text {a }}$ edic.).

9 Acerca de estos problemas puede verse, entre otros, el trabajo de P. KERESzTES, «The Decían libelli and Contemporary Literature», Latomus, 34 (1975), 761 y ss.

10 Sobre estos problemas remitimos, entre otros, a R. SYME, Emperors and Biography. Studies in the Historia Augusta, Oxford, 1971.

11 De ahi el calificativo de libeláticos asignado a los apóstatas cristianos por haber aceptado la firma de dichos escritos y todo lo que la misma implicaba desde el punto de vista religioso y político romano (no debemos olvidar que la religión romana era ante todo una religión política). 
el punto de vista del procedimiento administrativo-judicial romano parecen arrancar ya de algunas décadas anteriores, en concreto de la época de los Severos ${ }^{12}$.

Por su parte para comprender lo que pensaban, al menos en las primeras décadas del siglo III, los integrantes del círculo de intelectuales y jurisconsultos del Imperio, en especial quienes tenían su residencia en Roma, con respecto al cristianismo es posible afirmar que, al igual que sucedería en la centuria precedente, tal vez el punto clave continuaría siendolo la novedad que para ellos representaba la nueva religión en lo referente a las formas de vida y a los cultos tradicionales romanos ${ }^{13}$.

Frente a ello los apologistas cristianos, además de profundizar cada vez más en los rasgos que iban a definir la organización y jerarquización de la Iglesia, propugnarían continuos ataques contra las costumbres del Imperio heredadas de los antepasados, especialmente en el campo religioso, cuyo abandono pasaría a ser considerado como una provocación por parte de los ciudadanos romanos ${ }^{14}$.

La esperada reacción de los escritores paganos conduciría inexorablemente hacia un tipo de actuación violenta, que en un principio encontraría su base y fundamento en las masas populares de los centros urbanos más significativos.

De esta manera a lo largo de todo el siglo II y la primera mitad de la centuria siguiente tales personajes, como manifestación del sentir general de la intelectualidad romana, se mostrarían desconfiados y predispuestos negativamente contra el cristianismo, puesto que, además de que los adeptos de dicha religión no practicaban los cultos relacionados con las divinidades romanas ${ }^{15}$, los paganos les iban a achacar todo tipo de delitos

12 Sobre tales cuestiones remitimos a W. WILLIAMS, «The libellus Procedure and the Severan Papyri», JRS, 64 (1974), 86 y ss.

13 Para más detalles ver, entre otros, S. BENKO, «Pagan Criticism of Christianity during the First Two Centuries A.D.», ANRW, 2.23 .2 (1980), 1055 y ss.

14 Quizás tengamos que concretar en este punto el origen de las acciones de persecución contra las comunidades cristianas en su conjunto y contra algunos de los componentes de las mismas de forma individual. Cf. C.A. Contreras, "Christian Views of Paganism", ANRW, 2.23.2 (1980), 974 y ss.

15 Poniendo de manifiesto de esta manera su ateísmo en relación con el panteón romano. Cf. M.S. BuRRows, «Christianity in the Roman forum. Tertullian and the Apologetic Use of History», vChr, 42 (1988), 209 y y ss. 
y acusaciones infundadas (muertes, obscenidades) en conexión con las manifestaciones de su culto ${ }^{16}$.

Por su parte del lado cristiano hemos de destacar las referencias recogidas por los apologistas, aun cuando las puntualizaciones de estos autores cristianos difieren de las realizadas por sus colegas de la centuria precedente.

De esta manera, en un primer momento se hacía preciso afrontar el clima hostil de la sociedad romana y de los intelectuales paganos mediante la utilización de unos planteamientos filosófico-morales y retóricos que fueran inteligibles y admisibles a un mismo tiempo ${ }^{17}$.

Sin embargo, sería sólo en la segunda mitad del siglo "l cuando los primeros apologistas latinos, en concreto los africanos Minucio Félix y Tertuliano, adaptarían todos los conocimientos que poseían sobre la cultura latina a la defensa de las comunidades de los cristianos ${ }^{18}$.

En este sentido la personalidad y la actuación de Tertuliano constituirían el momento de inflexión, puesto que en sus obras encontraremos no sólo una defensa a ultranza del cristianismo, sino también una descripción más o menos detallada acerca de los avatares experimentados por los integrantes de dicha religión como consecuencia de las actividades emprendidas contra ellos por parte de las autoridades romanas ${ }^{19}$.

Además dicho escritor norteafricano llegaría a considerar al emperador Septimio Severo como el precursor de la actitud anticristiana de Decio, en realidad el primer gran perseguidor de la nueva doctrina religiosa, puesto que sus órdenes implicarian una política general contra los adeptos de dicha religión a lo largo y ancho de todas las provincias del Imperio ${ }^{20}$.

16 Por ello la apologética cristiana se vería obligada a hacer frente y defenderse de todas estas acusaciones. Ver, por ejemplo, J. SCHWARTZ, "Tradition and rupture dans l'apologétique chrétienne du Haut Empire», Ktèma, 10 (1985), 43 y ss.

17 En el fondo se trataría de demostrar que los cristianos eran compatibles con sus conciudadanos y con el régimen político con el que estaban obligados a convivir. $\mathrm{Cf}$. R. BEARE, "The Meaning of the Oath by the Safety of the Roman Emperors", AJPh, 99 (1978), 106 y ss., y R.M. Grant, Greek Apologists of the Second Century, Filadelfia, 1988.

18 N. SANTOS, Cristianismo e Imperio romano durante el siglo II, passium.

19 A. Quacquarelli, "La persecuzione secondo Tertulliano", Gregorianum, 31 (1950), 562 y ss.

20 P. Kenesztes, "The Emperor Septimius Severus: a Precursor of Decius», Historia, 19 (1970), 565 y ss. 
Con respecto a los pormenores de las acciones de persecución anticristiana del siglo III destaca la figura del historiador eclesiástico Eusebio de Cesarea, quien nos documenta claramente los pormenores de comportamiento desplegado por los emperadores de dicha centuria que se erigieron en perseguidores de los cristianos ${ }^{21}$.

De la misma manera en su descripción histórica, en parte heredada y similar a la que redactara Dionisio de Alejandría, se incluyen los ejemplos más representativos de los martirios que tuvieron su desarrollo en el transcurso de los reinados de dichos emperadores ${ }^{22}$.

Para completar esta visión contamos con las noticias contenidas en la documentación epistolar de Cipriano, obispo de Cartago, acerca de los diferentes vaivenes de las persecuciones del siglo III, incidiendo de forma especial en las acciones emanadas de los edictos imperiales de Decio, en cuyo caso admite la existencia de una violenta actividad de las masas populares, dirigida de forma especial contra los obispos y los clérigos del Norte de África ${ }^{23}$.

Por último no hemos de olvidar la importancia y alcance que, como fuente de información histórica, suponen los Acta o Vitae de los mártires cristianos (martirológios), a pesar de los inconvenientes cronológicos que se derivan de algunos de dichos documentos, sin desdeñar en ningún momento los interrogantes implicados en muchos casos con respecto a la época de su redacción.

A pesar de todo, un buen número de tales descripciones correspondientes a estos juicios y procesos contra los integrantes del cristianismo aparece confirmado en ocasiones en los autores anteriormente mencionados (Eusebio de Cesarea, Cipriano de Cartago...), por lo que su contenido histórico parece hallarse exento de cualquier duda.

En este contexto se enmarcan ias Vidas de quienes sufrieron el martirio en tiempos de Valeriano (entre otras las correspondientes a Mariano y Santiago; Fructuoso, Augurio y Eulogio; Montano, Lucio y algunos compa-

21 Sobre estas cuestiones remitimos a D.S. WALLACE-HADRILL, Eusebius of Caesarea, Londres, 1960, y A. DuMPE, Eusebios als Historiker, Munich, 1964.

22 Sobre estos aspectos cf. G. LAZZATTi, «Nota su Eusebio epitomatore di Atti di martiri», Studi Calderini-Paribeni, Milán, 1956, 1, pp. 377 y ss.

23 Ep. 7.1.8; 14.1.; 20.1; 59.6 y 66.4... Cf. P. KERESzTES, «The Decian libelli and Contemporary Literature», pp. 767-774. 
ñeros más) como consecuencia de los dos edictos anticristianos emitidos por dicho emperador y cuya cronología aparece claramente confirmada en comparación con la correspondencia epistolar de Cipriano por ejemplo ${ }^{24}$.

\section{EL CONTEXTO HISTÓRICO}

La historia de las comunidades cristianas a lo largo del siglo 111 , de la misma manera que sucedería desde sus orígenes y se prolongaría después hasta nuestros días, resulta inseparable del desarrollo político propio de aquella época y del marco socio-económico en que estaban arraigadas (en nuestro caso el Imperio romano a lo largo de los años que transcurren entre el 193 y el 284).

Como consecuencia de ello la evolución del cristianismo se comprende como un fenómeno inmerso en el contexto político, social e ideológico correspondiente a la evolución experimentada por el Estado romano en el transcurso de la etapa ocupada por los emperadores de la dinastía de los Severos y los propios de la Anarquía militar ${ }^{25}$.

No obstante, en algún momento sobrepasaremos esta fecha final de referencia, puesto que un cierto grupo de cristianos, cuyo martírio se fecha en los años inmediatos a la Gran Persecución de Diocleciano de comienzos del siglo $\mathrm{N}$, serían objeto de arresto y persecución como resultado de una normativa legal ya existente con anterioridad y no por obra de nuevos edictos o decretos debidos al fundador de la Tetrarquía ${ }^{26 .}$

Hemos analizado ya en otro lugar cómo la religión cristiana había encontrado unas instituciones estables a lo largo del siglo segundo de nuestra era, lo que había conducido a la búsqueda de la regulación de un funcionamiento uniforme en el interior de cada una de sus comunidades.

En otras palabras, se habían definido ya los principios básicos comunes que deberían ser aceptados por la totalidad de los adeptos del cris-

\footnotetext{
24 Sobre este particular remitimos, entre otros, a P. KERESZTES, «Two Edicts of the Emperor Valerian», vChr, 29 (1975), 81 y ss.

25 Ver, por ejemplo, a este respecto A. ALFöLDI, Studien zur Geschichte der Weltkrise des 3. Jahrhunderts n. Chr., Darmstadt, 1967.

${ }^{26}$ Sobre el cambio de política experimentado por dicho emperador con respecto a los cristianos remitimos a M.H. FRITZEN, Methoden der diocletianischen Christenvertolgung, Di y ss. Mainz
} 1961. 
tianismo, incluida una moral encaminada hacia la realización de buenas acciones en conexión con la vida cotidiana ${ }^{27}$.

Desde los inicios de dicha centuria se descubren los primeros atisbos de esta adaptación, que en última instancia no iba a mostrar una uniformidad absoluta en las diferentes regiones del Imperio romano sino que en cada caso se manifestaría como el resultado directo de la situación y de las tradiciones propias del territorio en que cada una de las comunidades cristianas se hallaba establecida.

Al mismo tiempo en las décadas finales de ese siglo II se produciría la consolidación de la estructura interna de la Iglesia cristiana como consecuencia de su adaptación a las instituciones político-administrativas y municipales romanas con las que estaba en contacto, tanto en el mundo oriental como en el occidental ${ }^{28}$.

Será entonces cuando surgirán los jefes de las distintas comunidades cristianas, cuyas prerrogativas sociales, espirituales, jurídicas y religiosas se iban a concentrar en el obispo ( $y$ en menor medida en los presbíteros y diáconos que se hallaban al frente de cada grupo o comunidad de cristianos), tomando como modelo y punto de referencia la organización característica de la monarquía imperial ${ }^{29}$.

Por ello la gran expansión alcanzada por la religión cristiana no sería más que el resultado inmediato de las condiciones políticas y económicas excepcionales de que disfrutaría el Imperio romano en el transcurso de las décadas correspondientes a la dinastía de los Antoninos, lo que incidiría a su vez de manera directa en la ampliación de las conexiones en la cuenca mediterránea a todos los niveles (relaciones comerciales, culturales y sociales) ${ }^{30}$.

De esta manera el cristianismo se expandiría en una primera fase por toda la península de Anatolia y los países de la cuenca del Egeo, para pasar a continuación a enraizarse en enclaves tan significativos como los

\footnotetext{
27 N. SANTOS, Cristianismo e Imperio romano durante el siglo 11 , passium.

28 Desde el punto de vista político romano hemos de remitir a F. JACQUES, Le privilège de liberté. Politique impériale et afltonomie municipale daos les cités de l Occident romain (161-244), Roma, 1984.

29 M. SACHOT, «Comment le christianisme est-il devenu religio?», RSR, 59 (1985), 95 y ss.

30 F. Bovon, «Pratiques missionaires et communication de f'Évangile dans le christianisme primitif", RThPh, 114 (1982), 369 y ss.
} 
centros urbanos de Antioquía en Siria y Alejandría en Egipto, donde se constata la presencia de comunidades cristianas cada vez más florecientes a medida que avanzamos en el tiempo ${ }^{31}$.

Por su parte el Occidente mediterráneo daría acogida igualmente en sus aglomeraciones urbanas a las agrupaciones más representativas de cristianos, que se convertirían al mismo tiempo en los núcleos difusores de la nueva doctrina sobre los territorios vinculados a ellas (dando lugar a la expansión por el ámbito rural).

Así pues, hemos de destacar no sólo la preeminencia lograda a este respecto por la capital del Imperio romano sino también el significado que alcanzarán algunas ciudades, como por ejemplo Cartago en el Norte de África ${ }^{32} \mathrm{o}$, ya desde la centuria precedente, Lugdunum (Lyon) en el ámbito territorial correspondiente a las provincias galas.

Ahora bien, será solamente a partir del siglo III cuando el latín se convertirá en el principal vehículo de transmisión de las creencias y prácticas cristianas, mientras que hasta ese momento lo sería el griego, por lo que no resulta difícil entender que la religión cristiana se vería saturada de reminiscencias e influencias helenísticas.

Este hecho marcaría precisamente las diferencias existentes entre las comunidades cristianas vinculadas a la Iglesia latina con centro en Roma y los grupos de adeptos cristianos asentados en la parte oriental del Imperio ${ }^{33}$.

De cualquier forma no hemos de exagerar, como ha ocurrido con bastante frecuencia hasta nuestros días por parte de ciertos investigadores, el número de cristianos que serían objeto de martirio a lo largo de la centuria que estamos analizando; en este sentido la documentación escrita que se fue configurando en torno a ellos (plasmada básicamente en los Acta martyrum correspondientes), se convertiría en el medio más útil y popular para pasar a ser el medio de propagación y propaganda de la nueva doctrina.

31 Como ejemplo nos pueden servir las referencias de R.M. GRANT, «Early Alexandrian Christianity", $\mathrm{CH}, 40$ (1971), 133 y ss.

32 G. SCHOELLGEN, «Die Teilnahme der Christen am städtischen Leben in vorkonstantinischen Zeit. Tertullians Zeugnis für Karthago», RQA, 77 (1982), 1 y ss.

${ }_{33}$ G. MussIES, "Greek as Vehicle of Early Christianity", NTS, 29 (1983), 356 y ss. 


\section{RELACIONES CRISTIANISMO-IMPERIO ROMANO}

Ante todo hemos de desechar la idea de que las comunidades de cristianos serían perseguidas sistemáticamente por parte de las autoridades romanas, incluidos los propios emperadores, a lo largo y ancho de todo el territorio del Imperio hasta el momento en que se produjo el reconocimiento oficial de la Iglesia en tiempos del emperador Constantino ${ }^{34}$.

Es por ello que el nivel y sentido de las relaciones establecidas entre el Estado romano y el cristianismo durante los años del siglo III se hallan envueltos en una acusada complejidad, en parte derivada de las peculiaridades y vaivenes experimentados por el poder político a lo largo de dicha época, acentuándose negativamente dicha situación en los 50 años correspondientes al período de la historia del Imperio romano conocido como Anarquía militar.

A este respecto podemos adelantar que los emperadores de la dinastía de los Severos (primer tercio de dicha centuria) continuarían vinculados a los parámetros propios de una política de actuación religiosa matizada, al igual que había sucedido en tiempos de los Antoninos ${ }^{35}$, por la racionalidad con respecto a los cristianos, lo que en parte al menos parece contrastar con la actuación de ciertos representantes de la administración romana en las provincias.

Sin embargo, la situación cambiaría en cierto sentido a medida que se inicia la época de crisis del siglo III, estando encabezada por Maximino el Tracio la primera fase de radicalización con respecto a los cristianos ${ }^{36}$.

A pesar de todo existe una tendencia bastante marcada en la historiografía de nuestro siglo a considerar que el cristianismo, al ser objeto de una persecución general y sistemática por parte de las autoridades e instituciones romanas, tanto a nivel de la administración central como pro-

34 En realidad esta situación de privilegio para los adeptos de dicha religión, y en especial para los jerarcas de sus comunidades, no se haría definitiva hasta el año 380 con el emperador Teodosio. Cf. G. EGGer, «Das Edikt des Kaisers Theodosius von 380 und das Ende der Konstantinischen Religionspolitik», Festschrift J.B. Trentini, Innsbruck, 1990, pp. 99 y ss.

35 J. BeAuJeu, «Religion in the Second Century A.D.", BR, 14.2 (1966), 1 y ss.

36 Ver como ejemplo, entre otros, los estudios de P. KERESzTES, «The Emperor Maximinus Decree of 235 A.D. Between Septimius Severas and Decius", Latomus, 28 (1969), 601 y ss.; G.W. ClaRKE, "Some Victims of the Persecution of Maximinus Thrax», Historia, 15 (1966), $445 \mathrm{y}$ ss.; y N. SANTOS, «Maximino el Tracio y los cristianos», Estudios Clásicos, 25 (1981-1983), 257 y ss. 
vincial o local (emperadores, gobernadores de provincias, procónsules, duunviros, prefectos de Roma, irenarcas...), aportaría una cantidad enorme de mártires entre sus adeptos durante las tres primeras centurias de nuestra era, a pesar de que un grupo de Actas de martirio asignadas a los mismos no resista una crítica histórica profunda ${ }^{37}$.

De esta manera, aun teniendo en cuenta la buena disposición de las instancias superiores de la administración romana del siglo $\|$ hacia las comunidades cristianas, dado que las autoridades políticas no parecen haberse erigido en ningún momento en obstáculo insalvable con vistas al proselitismo de los adeptos y prácticas de culto de dicha religión ${ }^{38}$, sin embargo encontrarian dificultades serias para consolidarse como una institución de carácter universal, así como para organizarse de acuerdo con los principios propios de una Iglesia plenamente estructurada ${ }^{39}$.

Por lo tanto es posible afirmar que la consolidación del cristianismo a lo largo de dicha centuria implicaría simultáneamente el desarrollo de los gérmenes de un conflicto inminente con las autoridades administrativas y gubernamentales del Imperio romano.

Esta situación acabaría por adquirir unas características de violencia a lo largo del siglo III, de manera especial en el transcurso de la persecución anticristiana llevada a cabo por parte del emperador Decio (y en una fase posterior por Valeriano) ${ }^{40}$.

En otros términos: a pesar de la situación favorable de los cristianos en tiempos de la dinastía de los Antoninos, permanecía latente un grave problema, consistente en el excesivo celo puesto de manifiesto por parte de los gobernadores y jueces provinciales, que sobrepasaba el cumplimiento

37 Sabemos a este respecto que hasta la época de Decio las órdenes emanadas de la cancillería imperial no abarcarían todo el territorio correspondiente al Imperio romano, por lo que no es de extrañar que sea en dicho período cuando el número de mártires cristianos aumente, aunque no hasta extremos desorbitados como en ocasiones se ha pretendido.

38 A pesar de que, bajo la presión de las masas populares, los representantes de la administración (prefecto de Roma, gobernadores provinciales, irenarcas, miembros de los senados municipales...) se verían obligados a afrontar cierto número de procesos contra los integrantes del colectivo de cristianos.

39 Será en su propio ámbito donde van a surgir discusiones y confrontaciones de tipo doctrinal y dogmático, dándose origen como consecuencia de ello a todo un conjunto de herejías (montanismo, gnosticismo, marcionismo...). Cf. R. TEJA, El cristianismo primitivo en la sociedad romana, Madrid, 1990, pp. 31-32.

40 Para más detalles remitimos a N. SANTOS, «Decio y la persecución de los cristianos», $M H A$, 15-16 (1994-1995), 143 y ss. 
estricto de la normativa emanada al respecto de la cancillería imperial. Como resultado de ello se llegaría a exigir de los adeptos de la religión cristiana una serie de libaciones y sacrificios en presencia de las estatuas de los emperadores ${ }^{41}$.

Y ello a pesar de que los cristianos, por boca de los apologistas, no se opondrían en ningún momento a elevar súplicas, en las oraciones dirigidas al Dios objeto de su culto, impetrando la salud del emperador respectivo en cada momento ${ }^{42}$.

Entre las Actas correspondientes a finales de dicho siglo, en las que sobresalen los primeros mártires del cristianismo africano en Scillitum ${ }^{43} \mathrm{o}$ la del senador Apolonio en Roma ${ }^{44}$, encontramos los elementos que sirven de base para dicha negativa, así como los argumentos utilizados para demostrar la lealtad de los adeptos de la nueva religión hacia la figura imperial y el poder que ésta representaba.

La conducta opuesta de los cristianos hacia manifestaciones de tal naturaleza pasaría a ser considerada, desde el punto de vista jurídico romano, como una actitud irreverente y contraria a los intereses políticos del Estado, por lo que tales actos serían incluidos en el concepto de crimen maiestatis (crimen de lesa majestad), a lo que contribuiría sin duda el empecinamiento puesto en escena por parte de las masas paganas de los centros urbanos ${ }^{45}$.

Ahora bien, este proceso de recrudecimiento en cuanto a las medidas tomadas por la administración se iría intensificando a lo largo del siglo III, primero a través de medidas destinadas a frenar el proselitismo de dichos cultos, y posteriormente mediante edictos orientados en toda su amplitud al resquebrajamiento de las estructuras en que se apoyaba la organización y funcionamiento de la Iglesia.

41 Sobre estos problemas remitimos, entre otros, a F. MILLAR, «The Imperial Culf and the Persecution", Le culte des souverains dans l'Empire romain, Vandoeuvres-Genève, 1973, pp.143 y ss.

42 R. BEARE, "The Meaning of the Oath by the Safety of the Roman Emperor», AJPh, 99 (1978), 106 y ss.

43 Ver, por ejemplo, N. SANTOS y M. GARCíA, «Los primeros mártires cristianos de la Iglesia africana», MHA, 15-16 (19941995), 291 y ss.

$44 \mathrm{M}$. SoRDI, «L'apologia del martire romano Apollonio come fonte dell'Apologeticum di Tertulliano e i rapporti fra Tertulliano e Minucio», RSCl, 18 (1964), 169 y ss. Cf. N. SANTOS y M. GARCiA, "Mártires cristianos del siglo Il», MHA, 13-14 (1992- 1993), 120.

45 M. SoRd, Los cristianos y el Imperio romano, Madrid, 1988, pp. 165-166. 
En el primero de los casos hemos de destacar algunos decretos orientados hacia la prohibición de catequizar a nuevos individuos con vistas a su ingreso en el seno del cristianismo, hecho que tendría su expresión más clara ya desde los primeros años de la dinastía de los Severos en la persona de Septimio Severo ${ }^{46}$.

Como elemento de enlace en la prosecución de estos dos objetivos contamos con la llamativa referencia de Eusebio de Cesarea en el sentido de que Maximino el Tracio daría orden de perseguir y ejecutar únicamente a los jefes de las iglesias cristianas (obispos, diáconos y presbiteros) porque eran responsables de la enseñanza de la doctrina cristiana; en este hecho se ha pretendido cifrar uno de los elementos que contribuirían a entender mejor las causas y motivos de las actividades anticristianas dirigidas por dicho emperador ${ }^{47}$.

Sin embargo, a partir de mediados del siglo III los objetivos imperiales iban a estar encaminados a desintegrar, mediante todas las medidas a su alcance, las bases que hacían posible que las comunidades de cristianos no sólo se ampliasen cada vez más por todo el Imperio sino también tratando de poner freno a la organización de la Iglesia, que con el paso del tiempo encontraba más apoyo entre los miembros de las clases elevadas de la sociedad romana con el consiguiente peligro para el propio Imperio ${ }^{48}$.

De cualquier forma es posible rastrear un hecho significativo: a pesar de tocia esta serie de trabas y cortapisas puestas de relieve por la administración romana, el siglo III iba a constituir, con relación a algunos aspectos al menos, una etapa de afianzamiento, consolidación y revitalización del cristianismo, considerado en el ámbito de la decadencia y crisis del Estado romano, así como de la cultura antigua en general a lo largo de dicha centuria.

Como resultado de ello los conflictos esporádicos (o en ocasiones sistemáticos, como los emprendidos por Decio, y en parte también por

46 SHA, Vita Sev. 17.1. Cf. J. SPEIGL, «Die Christenpolitik des Septimius Severus», MThZ, 20 (1969), 181 y ss.; y M. LEONE, «ll provvedimento di Settimio Severo contro i cristiani", Studi Manni, Roma 1980, pp.1317 y ss.

47 P. KERESzTES, «The Emperor Maximinus, Decree of 235 A.D.», p. 612.

48 En primer lugar sería Decio y posteriormente Valeriano quienes tratarian de cercenar y lograr la desintegración de la Iglesia cristiana mediante una serie de rescriptos emanados de las cancillerías imperiales. Cf. N. SANTOS, "Valeriano y la persecución de los cristianos", Espacio, Tiempo y Forma, Ser. II, Historia Antigua, 8 (1995), 197 y ss. Con respecto a la segunda fase ver P. KERESZTES, «Two Edicts of the Emperor Valerian», pp. 81 y ss. 
Valeriano) llevados a cabo contra la organización de la Iglesia no iban a impedir el florecimiento de amplios centros de cultura cristiana en enclaves como Alejandría, al tiempo que se produciría una nueva era de expansión de dicha religión, en especial en el marco de las provincias orientales del Imperio ${ }^{49}$.

Finalmente el emperador Galieno, con su actuación encaminada hacia el reconocimiento de la Iglesia, no haría más que valorar, desde la cúspide del poder romano, una realidad social que, a pesar de ciertos períodos de confrontación directa con la administración romana, iba aumentando cada vez más e incluyendo entre sus adeptos a grupos más numerosos de todas las clases sociales y administrativas del Imperio, tanto civiles como militares ${ }^{50}$.

En este sentido, por tanto, la política de Constantino con respecto al cristianismo ${ }^{51}$ no supondría más que el asentimiento oficial de algo que venía fraguándose ya a lo largo de todo el siglo Ill y que en el fondo implicaba la sanción a una ideología religiosa, cuyos valores culturales e importancia social hacían frente a las estructuras y formas tradicionales de vida antiguas (incluido el carácter político de la religión romana).

49 Para abundar en estos aspectos remitimos a N. SANTOS, El cristianismo en el marco de la crisis del Imperio romano en el siglo II, Oviedo, 1996.

50 N. SANTOS, "Galieno y la paz de la Iglesia", HAnt, 19 (1995), 281 y ss.

51 Y ello a pesar de que los integrantes de sus comunidades deberían afrontar previamente la prueba definitiva representada por la Gran Persecución de los primeros años del siglo IV con Diocleciano y Maximino Daia como protagonistas principales. 